\title{
Comparative rheologic investigations in a vertically rotating flume and a "moving-bed" conveyor belt flume
}

\author{
R. Kaitna, D. Rickenmann \& S. Schneiderbauer \\ Institute of Mountain Risk Engineering, BOKU University, Austria
}

\begin{abstract}
Debris flows present a serious hazard in mountainous regions. Though considerable research has been carried out to explain the movement of such heterogeneous mixtures of sediment, water, and air on a physical basis, rheologic models are still useful tools in engineering application. In order to study the flow behaviour of debris flow-like material, a vertically rotating flume as well as a conveyor belt-flume have been built. Both facilities allow to establish quasistationary conditions for an extended time period and to measure relevant flow parameters. Different mixtures of the synthetic polymer Carbopol Ultrez10 have been tested in the rotating flume as well as in the conveyor belt apparatus. Rheologic parameters of the Bingham model have been determined from drum data and from conveyor belt data for all mixtures following three different approaches. Independent measurements carried out in a conventional co-axial cylinder rheometer (Bohlin Visco 88) shows good agreement with the indirect determination of the rheologic parameters using the two experimental devices.
\end{abstract}

Keywords: Bingham model, rotating drum, conveyor belt flume, Carbopol.

\section{Introduction}

Debris flows are inherently complex and rare phenomena in mountain regions. Peak discharge and flow depth can be higher by an order of magnitude compared to flood events resulting from similar meteorological and hydrological conditions in the same catchment. High velocities and bulk densities ranging from 1.4 to 2.6 $\mathrm{t} / \mathrm{m}^{3}[1],[2]$ are the reason of the very destructive nature of such mass flows.

Depending on water content and the composition of sediments, various forms of flows have been observed [3]. Strong efforts have been made to explain the 
movement of such heterogeneous mixtures on a physical basis, but still simple rheologic models are useful tools in engineering application.

Most rheologic models are based a variation of following resistance law:

$$
\tau=\tau_{y}+f(\gamma)
$$

where $\tau=$ shear stress, $\tau_{y}=$ yield stress, and $\gamma=$ shear rate.

It can be seen that the shear stress in the flowing mixture depends on a yield stress that has to be overcome and a function of the shear rate. Considering granular flows with a wide grain size distribution flow resistance cannot be described by a single phase rheologic model due to complex grain-grain and grain fluid interactions [1], [4], [5], [6]. However, mudflows and debris flows containing a considerable amount of clay have been successfully modelled using a Herschel-Bulkley model [4], [5], which can be written as

$$
\tau=\tau_{y}+K \cdot \gamma^{n}
$$

with $K$ and the exponent $n$ as material parameter. In case of a Bingham fluid the exponent $n$ equals 1 and $K$ is defined as the (linear) Bingham viscosity $\eta$.

Determination of rheologic parameters in conventional rheometers are restricted to fluids and water - sediment suspensions including grain sizes smaller than about $1 \mathrm{~mm}$. Investigations with a homogeneous fluid have been carried out in a rotating drum and a conveyor belt flume to test the possibility to use these setups as large scale rheometer for fine grained slurry flows. In both cases it is possible to establish steady flow conditions at different energy levels (i.e. velocities) and to measure relevant flow parameter.

The objective of this study is to test whether it is possible to use either experimental setup as large scale rheometer for homogeneous fluids. Using simple analysis, Bingham parameters have been derived and compared with independent measurements in a standard rheometer (Bohlin Visco 88).

\section{Experiments}

\subsection{Vertically rotating drum}

The rotating drum has a diameter of $2.5 \mathrm{~m}$ (Figure 1(a)). The inner surface of the circumference is skimmed to avoid possible flow instabilities due to irregular bottom curvature. The channel section has a width of $0.45 \mathrm{~m}$. The rectangular cross-section is confined on one side by stainless steel, on the other side by acrylic glass to allow observations from the side. In order to avoid slip at the flume bottom the inner circumference is roughened by a synthetic $5 \times 5 \mathrm{~mm}$ mesh of approximately $1 \mathrm{~mm}$ height. Between the engine and the drum a torque flange is installed in order to record the torque brought up by the engine to keep the tested material flowing at constant velocity. Further the geometry of the surge is measured by ultra-sonic sensors. Four strain-gauge based force plates 
are installed at two locations at the circumference, measuring pair-wise normal stress and shear stress in the middle third of the cross section. Drum position (i.e. mean velocity of the surge) is registered by a sliced ring fixed at the axis and a static photo-electric sensor. Derivative with respect to time yields rotation velocity (i.e. mean velocity of the surge). Finally surface velocity is determined from tracer particles and digital video analysis. Details of the experimental setup and instrumentation can be found in Kaitna and Rickenmann [7]. The sample volume for the tests in the rotating flume lay between 30 and 45 litres.
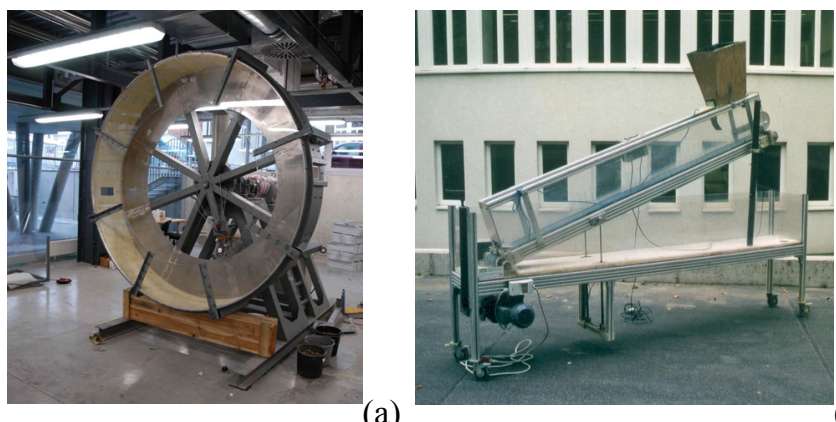

(a)

Figure 1: $\quad$ View of the drum (a) and the conveyor belt flume (b).

\subsection{Conveyor belt flume}

The conveyor belt apparatus (Figure 1(b)) has a channel length of $2.5 \mathrm{~m}$ and a width of $0.12 \mathrm{~m}$. The slope of the channel can be adjusted between $0^{\circ}$ and $30^{\circ}$, and has been varied for measurement in $2.5^{\circ}$ steps within the possible slope ranges. The volume of the tested material ranges between 4.0 and 4.5 litres.

Mean velocity is measured by an electronic speedometer directly attached to the conveyor belt, with an accuracy of $\pm 0.01 \mathrm{~m} / \mathrm{s}$. The inclination of the channel can be varied by hydraulic lever arm and has been determined with an accuracy of $\pm 0.15^{\circ}$. For each inclination, belt velocity was adjusted until a steady surge of approximately $3 / 4$ of the channel length was obtained. Flow depth was measured using a metering level at locations $20 \mathrm{~cm}$ and $60 \mathrm{~cm}$ behind the flow front. Repeated measurements revealed a reproducibility of about $1 \mathrm{~mm}$. Surface velocity was determined digitally by from top-view video recordings.

\subsection{Tested material and experimental procedure}

Investigations have been conducted with the synthetic polymer Carbopol Ultrez10®. It is a rheologic additive which modifies the shear resistance of water depending on concentration in the water. The flow curves can be described with a Herschel Bulkley model with a yield stress $\tau_{\mathrm{y}}>0$, and the exponent $\mathrm{n}<1$, i.e. viscoplastic flow behaviour. Technical datasheet of Carbopol Ultrez10® and details of rheologic behaviour can be found in Desai et al. [8], and Noveon [9].

The synthetic polymer has been produced some days in advance of the tests and stored in a closed container. Before each experiment the material was mixed 
using a hand drilling machine and then filled into the particular experimental apparatus. In case of "drum tests" the measurement system has been started and the speed of the drum increased. At each velocity, measurements have been performed during about 10 to 20 rotations and then averaged. Velocity was increased stepwise up to a speed where the flow visibly starts to become unstable and/or turbulent. For the "conveyor belt tests" the apparatus was started at the lowest inclination, then the material was filled in and conveyor belt speed was adjusted. After measurements were carried out, the slope angle was increased and the measurement procedure repeated.

During each state of constant speed in the drum and the conveyor-belt apparatus particles have been dropped repeatedly onto the surface of the flow for later particle tracking. Samples were taken from the reservoir before filling the drum, as well as before, during, and after each experimental run. The samples were analysed subsequently in a coaxial cylinder rheometer (Bohlin Visco $88 \mathrm{BV})$.

\section{Analysis and results}

\subsection{General observations}

Observations during the tests revealed that it is possible to establish stationary flow conditions in the rotating drum and in the conveyor belt apparatus within certain ranges of speed and inclination.

During the first tests in the rotating drum it became evident, that flow depth measurement by the ultrasonic sensor is restricted to the main section of the flow. Flow depth at the front and tail of the surge could not be measured directly by the employed system since the angle between direction of ultrasonic waves and flow surface at the front and the back region was too oblique for reception of the return-signal by the sensor. Assuming hydrostatic conditions the data from normal force plate measurement was converted into flow depth and compared with the data of the ultrasonic sensor for the overlapping region. The agreement between these independent measurements lies within $5 \%$ of total flow depth for all velocities. In the following all flow depth calculations are based on normal force measurements and checked with the partial data of the ultrasonic depth sensor.

Torque measurement at the axis of the drum can be compared with the deflection of the centre of gravity of the surge from the vertical. The torque calculated from surge geometry was generally lower than the torque measured directly, in most cases between 0 and $10 \%$. It was found that the main reason for this is that some material adheres to the drum along the entire circumference during rotation and thus does not contribute to the size of the "effective" surge; such "losses" have been reduced by a wiper mounted at the end of the surge to hinder material to be dragged away.

Flow depth measurements during the conveyor belt tests showed that the flow depth at location $20 \mathrm{~cm}$ behind the surge front and at location $60 \mathrm{~cm}$ differed mostly by less than $10 \%$. A mean value from these two measurements is 
considered representative for the surge and has been used in subsequent calculations.

Tests at high velocities and steep slopes at which the flow regime started to become unstable (onset of pulsing flow and roll-waves) have been excluded from further analysis.

Reynolds numbers were estimated for the experimental flows by introducing an "effective Newtonian viscosity", which has been calculated for each velocity as the ratio of mean boundary shear stress derived by measurement and shear rate estimated as outlined in Section 3.3. The values of Reynolds numbers for the drum experiments as well as conveyor belt tests are generally very low, ranging from 0.2 to 150 maximum. This is a clear indication of laminar flow regime during the tests. Froude numbers have been determined using mean flow depth. For both setups similar values between 0.2 and 4.4 have been obtained.

\subsection{Calculation of shear stress}

\subsubsection{Conveyor belt tests}

Estimation of mean bed shear stress is of main relevance for analysis. In case of the conveyor belt tests bed shear stress is directly derived from channel slope and flow depth measurement using the well known equation

$$
\tau_{0}=\rho \cdot g \cdot H \cdot \sin (a)
$$

where $\rho=$ density of the fluid, $g=$ acceleration due to gravity, $H=$ flow depth, and $\alpha=$ inclination angle of the channel.

\subsubsection{Rotating drum tests}

For the drum experiments boundary shear stress is derived from torque measurement (flange) and from calculation (deviation of centre of gravity). Assuming an equal distribution of bed shear stress and a triangular shear stress distribution on the side walls, mean shear stress of the surge is calculated with

$$
\tau_{\text {mean }}=\frac{T_{\text {net }}}{A_{B} \cdot R+2 \cdot \sum_{i=1}^{n}\left[\frac{1}{2} A_{S, i} \cdot\left(R-H_{i} / 3\right)\right]}
$$

where $T_{n e t}=$ net torque (friction losses due to the ball bearings are discounted), $A_{B}=$ wetted bottom area, $A_{S, i}=$ wetted partial area at the side, $R=$ radius of the drum, and $H_{i}=$ mean partial flow depth.

Shear stress resulting from equation (4) has been corrected to account for side wall friction following the procedure in Smart and Jäggi [10].

For further analysis of drum experiments an analogy is drawn to quasi-steady flow along a constant slope with angle $\alpha$ and flow depth $H$. The slope angle $\alpha$ used in the calculations is defined as the deflection angle of the centre of gravity from the vertical and the flow depth was defined as the mean flow depth of the total surge. The velocity at the flume bottom is assumed to be zero (no slip). This assumption seems to be justified since the inner surface of the circumference is 
roughened by a synthetic mesh. The assumption is supported by observations through the side wall of tracer particles that are dragged back with same speed like the circumference.

\subsection{Determination of Bingham model parameters}

In order to compare the data from the different experimental setups, the Bingham model was chosen. In the Bingham model the shear stress is given as

$$
\tau=\tau_{B}+\eta \cdot \gamma
$$

where $\tau_{B}$ and $\eta$ are Bingham yield stress and viscosity.

\subsubsection{Iterative strain rate estimation ("Iterative solution")}

In a first approach Bingham yield stress and Bingham viscosity are determined iteratively by assuming a linear velocity distribution within a sheared layer underneath an un-sheared "plug layer". Yield stress and viscosity can be estimated in a first step by dividing surface velocity by mean flow depth. In a next step a modified shear height is calculated by subtracting the plug height determined with $H_{\text {Plug }}=\tau_{B}{ }^{\prime} /(\rho . g . \sin \alpha)$ from mean flow depth. With the new shear rate a better estimate of yield stress and viscosity is obtained. This procedure is repeated iteratively.

\subsubsection{Strain rate estimation by assuming a parabolic velocity profile under an un-sheared region ("Parabolic solution")}

Assuming a parabolic velocity distribution underneath a plug flow, following equation for the strain rate is obtained [11]:

$$
\frac{d V}{d h}=\frac{2 \cdot V_{\text {plug }}^{2}}{3 \cdot V_{\text {surface }} \cdot H}
$$

where $d V / d h=$ shear rate, $V_{\text {plug }}=$ surface velocity relative to flume bottom, and $V_{\text {surface }}=$ surface velocity relative to laboratory floor.

In Figure 2 the results of shear rate estimates resulting from the "iterative solution" as well as the "parabolic solution" for all mixtures are plotted against the derived bed shear stress (dots). The solid and the dashed lines represent rheometer results for samples taken at the beginning of each experiment and at the end of each experiment, respectively. The decrease of viscosity is attributed to chemical pollution of the polymer during conveyor belt tests (a small amount of material is dragged with the conveyor belt out of the channel, gets in contact with rotating compounds of the apparatus and is delivered back into the channel). During the drum experiments no significant loss of viscosity was observed. It can be seen that following the "iterative solution" using data from drum experiments the rheometer curves can be very well reproduced. Error bars 
indicate a possible uncertainty of $10 \%$ of shear stress determination as explained in Section 3.2. Data from conveyor-belt tests show an increasing deviation from rheometer results with increasing shear rate. Here the error bars result from error propagation analysis. It is assumed that flow depth was measured with $\pm 1 \mathrm{~mm}$ accuracy and slope angle with $\pm 0.15^{\circ}$.
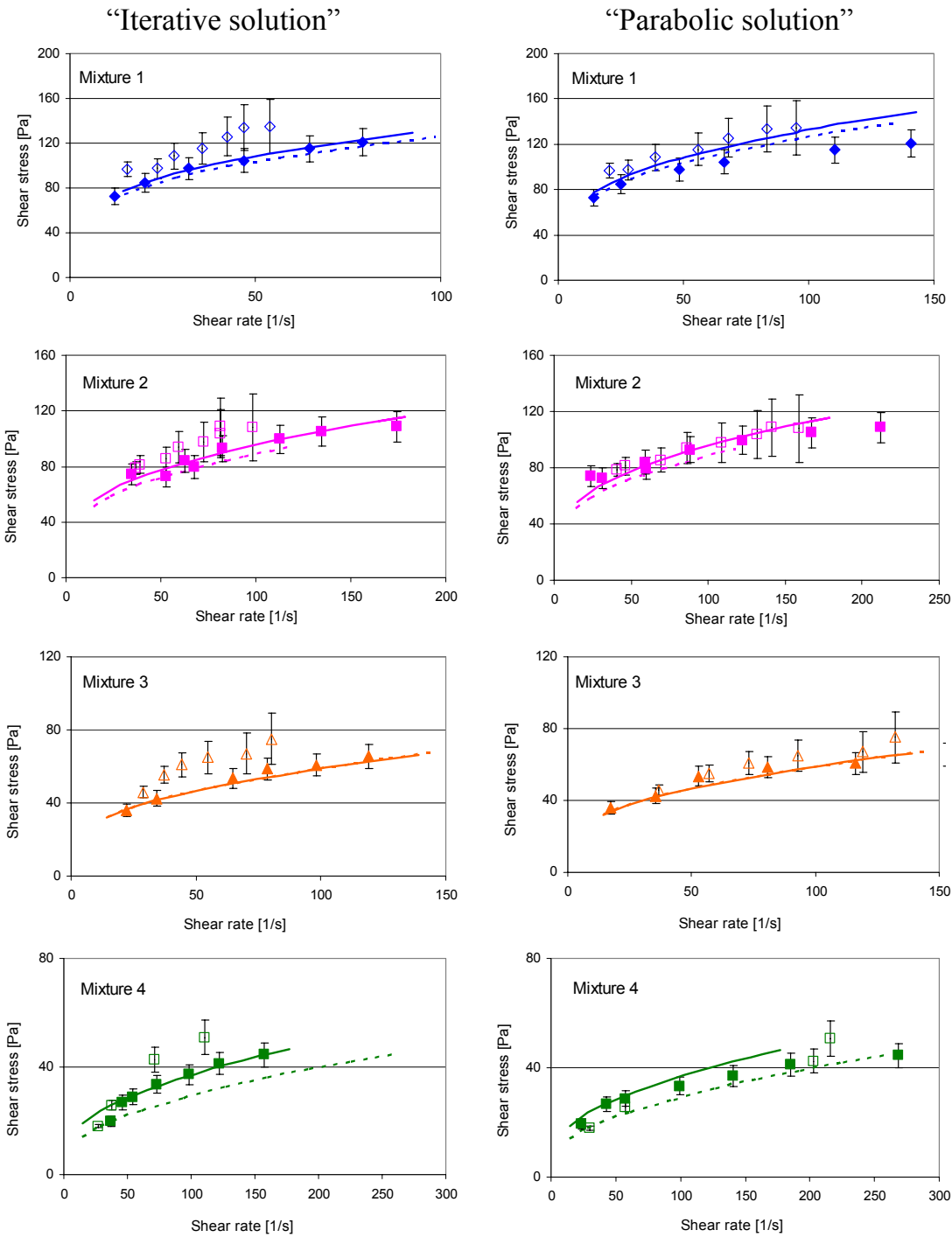

Figure 2: Rheograms of rheometer tests (solid and dashed line), drum experiments (full dots), and conveyor belt tests (open dots). 
When assuming a parabolic velocity profile ("parabolic solution") the shear rate estimates are generally higher than when a linear velocity profile is used ("iterative solution"), shifting the drum data as well as the conveyor belt data to the right. Conveyor belt data are now in better agreement with rheometer curves over the complete range of shear rates, but also drum data fits to rheometer in most cases.

\subsubsection{Depth averaged flow equation}

If equation (5) is applied to the laminar uniform flow of a Bingham fluid in an infinitely wide channel, the mean velocity in flow direction can be expressed as

$$
V_{m}=\frac{\tau_{0} \cdot H}{3 . \eta}\left[1-\frac{3}{2} \frac{\tau_{B}}{\tau_{0}}+\frac{1}{2}\left(\frac{\tau_{B}}{\tau_{0}}\right)^{3}\right]
$$

Bingham parameters of each mixture have been back-calculated from equation (7) using all possible combinations of pairs of datasets separately for rotating drum and conveyor belt experiments.

Figure 3: shows an overview of derived Bingham parameters from drum data and conveyor belt data as well as results from the independent rheometer measurement.
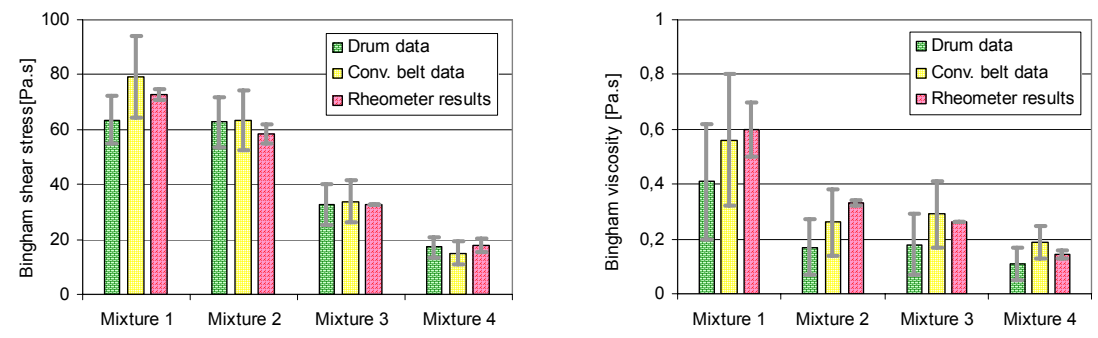

Figure 3: Comparison of results for Bingham shear stress (left) and Bingham viscosity (right).

Back calculation of Bingham shear stress was quite successful for both, data from drum experiments and from conveyor belt tests. The agreement with the rheometer results lies in nearly all cases within the limits of standard deviation of the single results. In the left diagram of Figure 3 it can be seen that backcalculated Bingham viscosity from drum data underestimates viscosity measured in the rheomter. Despite a large scatter for the viscosity results from conveyor belt tests, there is a very good agreement between measured and back-calculated Bingham viscosity. 


\section{Discussion and conclusion}

The analysis introduced herein is based on the postulation of a Bingham model which is applied to an inherent viscoplastic fluid - Carbopol Ultrez10®. The derived values of Bingham parameters are adjusted values; they are strictly valid only for the range of shear rate investigated, which might differ between experiments and rheometer measurement. The main uncertainty in rheologic analysis of non-Newtonian open channel flows is the derivation of a representative shear rate without ex ante knowing the exact material behaviour. For this reason a plug flow and a linear velocity distribution of the sheared layer (i.e. a rough estimation of real sheas rate) was postulated in a first approach. It is shown that for flows in a rotating drum this assumption leads to satisfying results. A better agreement with reference measurements for the data from conveyor belt test is achieved when assuming a parabolic velocity profile underneath a plug flow. Although a rheologic analysis should not be predicated on an assumed rheological behaviour [12], a more concave velocity distribution (resulting in higher shear rate estimates for the conveyor belt channel) is to be expected for a lot of artificial and natural (mud) flows [3], [5]. In case of the rotating drum tests it was found that shear rate estimates do not differ significantly resulting either from the linear or parabolic velocity-distribution assumption. The reason might be the non-uniform flow depth along the surge (in flow direction) due to a curved flow path, resulting in a varying velocity distribution (and shear rate). However, when conducting a simple global analysis of the flow, the simplifications outlined in Section 3.2 are found to be acceptable. Results from the depth averaged flow equation gives further confirmation of applicability of the experimental methods. Back-calculation of Bingham yield stress and viscosity using the depth averaged flow equation was successful for conveyor belt data, and for the data from drum experiments the yield stress calculations led to satisfying results, whereas viscosity was underestimated in two cases.

Four different mixtures of the synthetic polymer Carbopol Ultrez10® have been tested in a vertically rotating drum and a conveyor belt flume. Simple rheologic analyses were used the possibility to deduce the general flow behaviour from both setups. We found that it is possible to determine flow curves and Bingham parameters for single phase non-Newtonian fluids within the limits of accuracy. For hazard assessment, Bingham parameters are often needed as input parameters for numerical simulation models of debris flows [13].

\section{References}

[1] Iverson, R.M., Physics of debris flows. Reviews of Geophysics, 35(3), pp.245-296, 1997.

[2] Costa, J.E., Physical Geomorphology of Debris Flows (Chapter 9). Developments and Applications of Geomorphology, eds. J.E. Costa, P.J. Fleisher, Springer Verlag: Berlin and Heidelberg, pp. 268-317, 1984. 
[3] Hungr, O., Evans, S.G., Bovis, M.J., Hutchinson, J.N., A Review of the Classification of Landslides of the Flow Type. Environmental and Engineering Geoscience 7(3), pp.221-238, 2001.

[4] Coussot, P., Mudflow rheology and dynamics. IAHR Monograph Series, Balkema: Rotterdam, 1997.

[5] Parson, J.D., Whipple, K.X., Simoni, A., Experimental study of the grainflow, fluid-mud transition in debris flows. The Journal of Geology, 109, pp.427-447, 2001.

[6] Hungr, O., Analysis of debris flow surges using the theory of uniformly progressive flow. Earth Surf. Process. Landforms 25, pp. 483-495, 2000.

[7] Kaitna, R., Rickenmann, D., A new experimental facility for laboratory debris flow investigation. In preparation.

[8] Desai, D.D., Hasman, D.F., Schmucker-Castner, J.F. (1998). Advances in Carbomer Polymer Technology. In Cosmetics and Toiletties Manufacture Worldwide. Online. http://www.homecare.noveoninc.com

[9] Noveon (1995). Carbopol Ultrez10 polymer for personal care applications. Datasheet. Online. http://www.homecare.noveoninc.com

[10] Smart, G.M., Jäggi, M., Sediment transport on steep slopes. Mitteilung Nr. 64, Versuchsanstalt für Wasserbau Hydrologie und Glaziologie der Eidgenössischen Technischen Hochschule Zürich., 1983.

[11] Holmes, R.R., Huizinga, R.J., Brown, S.M., Jobson, H.I., Laboratory procedures and data reduction techniques to determine rheologic properties of mass flows. USGS Water-Resources Investigation Report, 93-4123, 1993.

[12] Major, J.J., Discussion: Verification of vertically rotating flume using non-Newtonian fluids. Journal of Hydraulic Engineering 123(10), pp.936937, 1997.

[13] Rickenmann, D., Laigle, D., McArdell, B. W., Hübl. J., Comparison of 2D debris-flow simulation models with field events. Computational Geosciences, in press, 2005. 